\title{
A Novel Extrapolation-Based Grey Prediction Model for Forecasting China's Total Electricity Consumption
}

\author{
Xin-bo Yang $\mathbb{D}^{1,2}$ \\ ${ }^{1}$ School of Economics and Business Administration, Chongqing University, Chongqing 400030, China \\ ${ }^{2}$ College of Mathematics and Information Engineering, Chongqing University of Education, Chongqing 400065, China
}

Correspondence should be addressed to Xin-bo Yang; yangxinbo0102@163.com

Received 10 February 2021; Revised 20 April 2021; Accepted 26 May 2021; Published 8 June 2021

Academic Editor: Xin Ma

Copyright $(2021$ Xin-bo Yang. This is an open access article distributed under the Creative Commons Attribution License, which permits unrestricted use, distribution, and reproduction in any medium, provided the original work is properly cited.

Accurately forecasting China's total electricity consumption is of great significance for the government in formulating sustainable economic development policies, especially, China as the largest total electricity consumption country in the world. The calculation method of the background value of the $\operatorname{GM}(1,1)$ model is an important factor of unstable model performance. In this paper, an extrapolation method with variable weights was used for calculating the background value to eliminate the influence of the extreme values on the performance of the $\operatorname{GM}(1,1)$ model, and the novel extrapolation-based grey prediction model called $\operatorname{NEGM}(1,1)$ was proposed and optimized. The $\operatorname{NEGM}(1,1)$ model was then used to simulate the total electricity consumption in China and found to outperform other grey models. Finally, the total electricity consumption of China from 2018 to 2025 was forecasted. The results show that China's total electricity consumption will be expected to increase slightly, but the total is still very large. For this, some corresponding recommendations to ensure the effective supply of electricity in China are suggested.

\section{Introduction}

With the rapid growth of the Chinese economy, the total electricity consumption is increasing year by year, and China is the largest energy consumer in the world currently. From China Statistical Yearbook 2019 [1], China's total electricity consumption reached 6282.1 billion kilowatt-hours (billion $\mathrm{kw} / \mathrm{h}$ ) in 2017; compared with 2016 and 2007, the electricity consumption increased by $4.67 \%$ and $98.16 \%$, respectively. Due to the increasing demand for electricity consumption in China, vigorously developing and efficiently using electricity to ensure the effective supply of energy is the inevitable choice in China. Therefore, the establishment of a corresponding mathematical model for accurately forecasting future electricity consumption is an important contribution to the formulation of China's energy security strategy and sustainable development program.

Since the end of the last century, numerous models have been introduced for forecasting electricity consumption, such as the time series analysis model [2,3], autoregressive integrated moving average (ARIMA) model $[4,5]$, support vector machine (SVM) [6, 7], Bayesian statistics [8], random forest [9], composite system method [10], artificial neural networks (ANNs) [11, 12], and deep learning models $[13,14]$. However, these exhibit the limitations of requiring a great many high-quality data samples.

The grey system theory, proposed by Professor Deng [15] in 1982, plays a key role in processing uncertain problems with poor information and small samples. After more than 30 years of development, many scholars have applied it to industry, agriculture, society, economy, energy, and other fields [16-20]. The prediction problem of total electricity consumption is a complex uncertainty problem, which can be thought of as a grey system problem. On the one hand, electricity consumption is closely related to a series of uncertain factors, such as the degree of economic development, industrial structure, population, and distribution losses; on the other hand, as China's statistical departments did not release annual data relating to the electricity consumption before 2000 years ago, the sample size is limited. However, the grey forecasting model has a good effect on such a "small sample, poor information" system. 
The grey forecasting model is an important component of grey system theory, which has attracted attention to improving the stability of simulation performance and the reliability of prediction results by many scholars. The traditional $\operatorname{GM}(1,1)$ model [16] is the most significant constituent of grey prediction. However, it can only simulate homogeneous exponential growth sequences. The main reason is that it is difficult for a sequence to completely satisfy the law of homogeneous exponential growth. Many studies were conducted on the improvement of the grey model mainly on the following four aspects: (1) converting the raw data $X^{(0)}$ for improvement of smoothness by some buffer operators and effective methods [21, 22]; (2) enhancing the computational methods of parameters by some intelligence algorithms to optimize the initial value and background value $[23,24]$; (3) extending the structure of traditional grey models $[25,26]$ for improving traditional model compatibility with different data, such as non-homogeneous sequence or fluctuation sequence; and (4) combining the grey prediction models with other models, such as combining neural network and Markov model with the grey prediction model $[27,28]$.

The background value is an important consideration of the grey model [29]. Currently, the background value is usually taken as the mean value of $x^{(1)}(k)$ and $x^{(1)}(k-1)$, which is defined as $z^{(1)}(k)=0.5\left(x^{(1)}(k)+x^{(1)}(k-1)\right)$. Geometrically, the trapezoidal area with a straight edge is used instead of the trapezoidal area with a curved edge. This is the main cause of the low prediction accuracy of the model. Many scholars have put forward methods to improve the traditional method of calculation of grey model background value to increase modeling accuracy. These calculation methods can be roughly divided into two types: one is to define the background value as $z^{(1)}(k)=\alpha x^{(1)}(k)+(1-$ $\alpha) x^{(1)}(k-1)$ [30]. The parameter $\alpha$ is an unknown interpolation coefficient, $\alpha \in[0,1]$, which is designed to adjust the background value to enhance the performance of grey forecasting model by some intelligence algorithms. The other one is to reconstruct the background value $z^{(1)}(k)$ by function integral [31] or numerical calculation method [32]. The above research studies on the background value greatly enriched the theoretical system of the grey prediction models. However, there exists a defect that the interpolation method was used to calculate the background value of these models. It can be determined from the above structures of background value that the extreme values $x^{(1)}(k)$ and $x^{(1)}(k-1)$ are important factors that affect the smoothness of the background value and even affect the performance of the model. Therefore, it is necessary to expand the calculating range of the background value to weaken the influence of extreme values on $z^{(1)}(k)$.

Inspired by the literature [33], a novel extrapolation method for calculating the background value of the grey forecasting model $(\operatorname{NEGM}(1,1))$ is proposed to predict China's total electricity consumption in this study. The calculation process of background value for the new model is composed of three values with variable weights $\alpha_{1} x^{(1)}(k)$, $\alpha_{2} x^{(1)}(k-1)$, and $\alpha_{3} x^{(1)}(k-2)$. Compared with the traditional grey models, $\operatorname{NEGM}(1,1)$ not only can significantly improve the smoothness effect of the background value but also can effectively weaken the influence of extreme values on the model's performance. In addition, some classic grey models are included in $\operatorname{NEGM}(1,1)$, and then, it can simulate the homogeneous exponential sequence, non-homogeneous exponential sequence, and linear function sequence unbiased.

As compared with the existing research, this paper is unique in the following two aspects:

(i) A novel grey forecasting model, $\operatorname{NEGM}(1,1)$, is proposed, which solves the problem that many grey prediction models cannot overcome the influence of extreme values and the data type is single.

(ii) The total electricity consumption in China is simulated and predicted by using the $\operatorname{NEGM}(1,1)$ model, and the measures to ensure the effective supply of electricity in China are put forward.

The rest of the paper is arranged as follows. We propose a novel grey prediction model $\operatorname{NEGM}(1,1)$ in Section 2. Section 3 presents modeling the evaluation criteria. In Section 4, the $\operatorname{NEGM}(1,1)$ model is used to compare simulation and errors with the other two grey models and predict the total electricity consumption of China. In Section 5 , countermeasures and suggestions are put forward based on the prediction results of Section 4. Conclusions are drawn in Section 6.

\section{The Novel Extrapolation-Based Grey Prediction Model}

In this section, the novel extrapolation-based grey prediction model called $\operatorname{NEGM}(1,1)$ is proposed and optimized. The modeling mechanism and prediction function of the new model are deduced.

Definition 1 (see [16]). Assume that a non-negative raw sequence is $X^{(0)}=\left(x^{(0)}(1), x^{(0)}(2), \ldots, x^{(0)}(n)\right)$, where $x^{(0)}(k) \geq 0$, for $k=1,2, \ldots, n . X^{(1)}$ is called the 1 -AGO (Accumulating Generation Operator) sequence of $X^{(0)}$, i.e.,

$$
X^{(1)}=\left(x^{(1)}(1), x^{(1)}(2), \ldots, x^{(1)}(n)\right),
$$

where

$$
x^{(1)}(k)=\sum_{i=1}^{k} x^{(0)}(i), \quad k=1,2, \ldots, n
$$

Definition 2. Assume that $X^{(0)}$ and $X^{(1)}$ are the same as in Definition 1 and $0 \leq \alpha_{i} \leq 1(i=1,2,3)$ are constants; when $k=3,4, \ldots, n$, the equation

$$
\begin{aligned}
& x^{(0)}(k)+a\left(\alpha_{1} x^{(1)}(k)+\alpha_{2} x^{(1)}(k-1)+\alpha_{3} x^{(1)}(k-2)\right) \\
&= k b+c, \\
& \cdot \alpha_{1}+\alpha_{2}+\alpha_{3}=1,
\end{aligned}
$$


is the novel extrapolation-based grey model with a single variable and one first-order equation, $\operatorname{NEGM}(1,1)$ for short.

According to the inverse process of Accumulating Generation Operator in Definition 1,

$$
x^{(0)}(k)=x^{(1)}(k)-x^{(1)}(k-1), \quad k=3,4, \ldots, n .
$$

There are some special cases of the $\operatorname{NEGM}(1,1)$ model as follows:

(i) When $\alpha_{1}=\alpha_{2}=0.5$ and $\alpha_{3}=0$ in equation (3), the $\operatorname{NEGM}(1,1)$ model reduces to the SAIGM model [34] with the following form:

$$
\begin{aligned}
x^{(0)}(k)+a z^{(1)}(k) & =k b+c, \\
z^{(1)}(k) & =\frac{1}{2}\left(x^{(1)}(k)+x^{(1)}(k-1)\right) .
\end{aligned}
$$

(ii) Setting $c=0$ in equation (5), the SAIGM model reduces to the $\operatorname{NGM}(1,1, \mathrm{k})$ model [35] with the following form:

$$
\begin{aligned}
x^{(0)}(k)+a z^{(1)}(k) & =k b, \\
z^{(1)}(k) & =\frac{1}{2}\left(x^{(1)}(k)+x^{(1)}(k-1)\right) .
\end{aligned}
$$

(iii) Setting $b=0$ in equation (5), the SAIGM model reduces to the classic $\operatorname{GM}(1,1)[16]$ with the following form:

$$
\begin{aligned}
x^{(0)}(k)+a z^{(1)}(k) & =c, \\
z^{(1)}(k) & =\frac{1}{2}\left(x^{(1)}(k)+x^{(1)}(k-1)\right) .
\end{aligned}
$$

It is easy to verify that the $\operatorname{NEGM}(1,1)$ model can unbiasedly simulate the homogeneous exponential sequence, non-homogeneous exponential sequence, and linear function sequence according to the above structural features.

Let the set of data sequence $X^{(0)}$ and $X^{(1)}$ be the same as in Definition 1; the background value is written as $z^{(1)}(k)=$ $\alpha_{1} x^{(1)}(k)+\alpha_{2} x^{(1)}(k-1)+\alpha_{3} x^{(1)}(k-2), \alpha_{1}+\alpha_{2}+\alpha_{3}=1$, $k=3,4, \ldots, n$. Then, the least-squares estimation for $\widehat{p}=(a, b, c)^{T}$ of the $\operatorname{NEGM}(1,1)$ model satisfies

$$
\widehat{p}=(a, b, c)^{T}=\left(B^{T} B\right)^{-1} B^{T} Y,
$$

where

$$
\begin{aligned}
& B=\left[\begin{array}{ccc}
-z^{(1)}(3) & 3 & 1 \\
-z^{(1)}(4) & 4 & 1 \\
\vdots & \vdots & \vdots \\
-z^{(1)}(n) & n & 1
\end{array}\right], \\
& Y=\left[\begin{array}{c}
x^{(0)}(3) \\
x^{(0)}(4) \\
\vdots \\
x^{(0)}(n)
\end{array}\right] .
\end{aligned}
$$

According to Definition 2 and equation (3),

$$
\widehat{x}^{(0)}(k)=\widehat{x}^{(1)}(k)-\widehat{x}^{(1)}(k-1), \quad k=3, \ldots, n .
$$

Then, we have

$$
\begin{aligned}
\hat{x}^{(1)}(k)-\hat{x}^{(1)}(k-1) & \\
& +a\left(\alpha_{1} \widehat{x}^{(1)}(k)+\alpha_{2} \widehat{x}^{(1)}(k-1)+\alpha_{3} \widehat{x}^{(1)}(k-2)\right) \\
= & k b+c .
\end{aligned}
$$

That is,

$$
\begin{aligned}
& \left(1+a \alpha_{1}\right) \widehat{x}^{(1)}(k)-\left(1-a \alpha_{2}\right) \widehat{x}^{(1)}(k-1) \\
& +a \alpha_{3} \widehat{x}^{(1)}(k-2)=k b+c .
\end{aligned}
$$

Arranging equation (12), we get

$$
\begin{aligned}
\hat{x}^{(1)}(k)= & \frac{1-a \alpha_{2}}{1+a \alpha_{1}} \hat{x}^{(1)}(k-1)-\frac{a \alpha_{3}}{1+a \alpha_{1}} \hat{x}^{(1)}(k-2) \\
& +\frac{b}{1+a \alpha_{1}} k+\frac{c}{1+a \alpha_{1}} .
\end{aligned}
$$

Let

$$
\begin{aligned}
& \delta_{1}=\frac{1-a \alpha_{2}}{1+a \alpha_{1}}, \\
& \delta_{2}=-\frac{a \alpha_{3}}{1+a \alpha_{1}}, \\
& \delta_{3}=\frac{b}{1+a \alpha_{1}}, \\
& \delta_{4}=\frac{c}{1+a \alpha_{1}} .
\end{aligned}
$$

Then, we get

$$
\begin{aligned}
\hat{x}^{(1)}(k)= & \delta_{1} \widehat{x}^{(1)}(k-1)+\delta_{2} \widehat{x}^{(1)}(k-2) \\
& +\delta_{3} k+\delta_{4}, \quad k=3, \ldots, n,
\end{aligned}
$$

and equation (15) is called the unbiased estimation form of the $\operatorname{NEGM}(1,1)$ model. According to Definition 1, the final restored expression $\hat{x}^{(0)}(k+1)$ is as follows:

$$
\begin{aligned}
\hat{x}^{(0)}(k+1)= & \widehat{x}^{(1)}(k+1)-\hat{x}^{(1)}(k) \\
= & \delta_{1} \hat{x}^{(1)}(k)+\left(\delta_{2}-\delta_{1}\right) \hat{x}^{(1)}(k-1) \\
& -\delta_{2} \hat{x}^{(1)}(k-2)+\delta_{3} .
\end{aligned}
$$

Because equation (15) is a second-order difference equation, it is complicated to deduce its time response function by the direct substitution method. In addition, since we only make a short-term prediction of China's total electricity demand, we can directly calculate $\hat{x}^{(1)}(k)$ and $\hat{x}^{(0)}(k)$ by equations (15) and (16). Notice that the main purpose of constructing the $\operatorname{NEGM}(1,1)$ model is to simulate or forecast $\hat{x}^{(1)}(k)$ and $\hat{x}^{(0)}(k)$, and the time response function of the NEGM $(1,1)$ model is not important for us. 


\section{Optimization of System Parameters}

The performance of one prediction model is evaluated by its simulation and prediction errors. To test the performance of $\operatorname{NEGM}(1,1)$, the mathematical meanings of symbols in Table 1 are defined as follows:

(i) $\hat{x}^{(0)}(k)$ is the simulation or prediction data of the real data $x^{(0)}(k)$.

(ii) $\Delta_{S}(k)$ is the relative simulation percentage error of $\widehat{x}^{(0)}(k)(\mathrm{RSPE}): \Delta_{S}(k)=\left(\left|\widehat{x}^{(0)}(k)-x^{(0)}(k)\right| / x^{(0)}\right.$ $(k)) \times 100 \%$.

(iii) $\Delta_{P}(k)$ is the relative prediction percentage error of $\widehat{x}^{(0)}(k)(\mathrm{RPPE}): \Delta_{P}(k)=\left(\left|\widehat{x}^{(0)}(k)-x^{(0)}(k)\right| / x^{(0)}\right.$ $(k)) \times 100 \%$.

(iv) $\bar{\Delta}_{S}$ is the mean relative simulation percentage error (MRSPE): $\bar{\Delta}_{S}=\sum_{k=1}^{n} \Delta_{S}(k) / n$.

(v) $\bar{\Delta}_{P}$ is the mean relative prediction percentage error (MRPPE): $\bar{\Delta}_{P}=\sum_{k=1}^{n} \Delta_{P}(k) / n$.

(vi) $\bar{\Delta}$ is the comprehensive mean relative percentage error (CMRPE): $\bar{\Delta}=0.5 \times\left(\bar{\Delta}_{S}+\bar{\Delta}_{P}\right)$.

When using the $\operatorname{NEGM}(1,1)$ model to forecast the original data, we first need to determine the parameters $\alpha_{i}(i=1,2,3)$ of the new model and then use equations (8) and (14) to obtain the parameters $(a, b, c)$ and $\delta_{i}(i=1,2,3,4)$, respectively, and the prediction values $\hat{x}^{(1)}(k)$ can be calculated by equation (15). In summary, the following minimum problem of the square of MRSPE can be defined to obtain the optimal parameters $\alpha_{i}(i=1,2,3)$.

$$
\begin{aligned}
& \min _{\alpha_{1}, \alpha_{2}, \alpha_{3}} \frac{1}{n-2} \sum_{k=3}^{n}\left|\frac{x^{(0)}(k)-\widehat{x}^{(0)}(k)}{x^{(0)}(k)}\right|^{2} \times 100 \%, \\
& \text { s.t. }\left\{\begin{array}{l}
(a, b, c)^{T}=\left(B^{T} B\right)^{-1} B^{T} Y, \\
\alpha_{1}+\alpha_{2}+\alpha_{3}=1, \\
0 \leq \alpha_{1}, \alpha_{2}, \alpha_{3} \leq 1 .
\end{array}\right.
\end{aligned}
$$

Since problem (17) is the convex quadratic programming problem, the optimal values of $\alpha_{i}(i=1,2,3)$ can be computed by the nonlinear solver of MATLAB. Figure 1 gives the calculation flowchart from which we can clearly understand the modeling process.

\section{Forecasting China's Total Electricity Consumption}

To verify the effectiveness of the $\operatorname{NEGM}(1,1)$ model in Section 3, we establish grey models for the total electricity consumption in China from 2000 to 2017 in Table 2. First, the original data to use in the empirical analysis are divided into two parts. The first part (years 2000-2015) is the training data used for modeling, and the other part (years 2016-2017) is used as a test set. In addition, two competing models, including SAIGM [34] and traditional $\operatorname{GM}(1,1)[16]$, are employed for simulating and forecasting the total electricity consumption in China. Finally, the model which has the best performance is used to predict future values from 2018 to 2025.

From Table 2,

$$
\begin{aligned}
X^{(0)}= & \left(x^{(0)}(1), x^{(0)}(2), x^{(0)}(3), x^{(0)}(4), \ldots, x^{(0)}(16)\right) \\
= & (1347.2,1463.4,1633.2,1903.2,2197.1,2858.9, \\
& \cdot 3271.2,3454.1,3703.2,4193.5,4700.1,4976.3, \\
& \cdot 5420.3,5638.4,5802.0) .
\end{aligned}
$$

The detailed modeling process of the $\operatorname{NEGM}(1,1)$ model contains four steps: parameter estimation, model construction, model performance test, and data prediction.

Step 1. Parameter estimation.

From Definition 1,

$$
\begin{aligned}
X^{(1)}= & \left(x^{(1)}(1), x^{(1)}(2), x^{(1)}(3), x^{(1)}(4), \ldots, x^{(1)}(16)\right) \\
= & (1347.2,2810.6,4443.8,6347,8544.1,11038.1, \\
& \cdot 13897,17168.2,20622.3,24325.5,28519,33219.1, \\
& \cdot 38195.4,43615.7,49254.1,55056.1) .
\end{aligned}
$$

The parameter sequence $\widehat{p}=(a, b, c)^{T}$ can be calculated by equation (8), and the unknown parameters $\alpha_{i}(i=1,2,3)$ of $\operatorname{NEGM}(1,1)$ are determined by the constraint optimization problem (17). The parameters of the $\operatorname{NEGM}(1,1)$ model are shown in Table 3.

Step 2. Model construction.

According to Table 3 and equation (14), the modeling parameters $\delta_{1}, \delta_{2}, \delta_{3}$, and $\delta_{4}$ are $1.0088,0.0006,305.0882$, and 633.0891, respectively. We obtain

$$
\begin{aligned}
\hat{x}^{(1)}(k)= & 1.0088 \hat{x}^{(1)}(k-1)+0.0006 \hat{x}^{(1)}(k-2) \\
& +305.0882 k+633.0891,
\end{aligned}
$$

and $k=3,4, \ldots, n$. Equation (20) is just the unbiased estimation form of the $\operatorname{NEGM}(1,1)$ model for forecasting the total electricity consumption in China.

Step 3. Model performance comparisons and tests.

The $\operatorname{NEGM}(1,1)$ model can be constructed and the simulation and prediction data of the $\operatorname{NEGM}(1,1)$ can be computed. Then, the relative simulation/prediction percentage errors (RSPE/RPPE), the mean RSPE/RPPE (MRSPE/MRPPE), and the comprehensive mean relative percentage errors (CMRPE) can be obtained from the simulation and prediction data. The calculation results are shown in Table 1.

As shown in Table 1 , the proposed $\operatorname{NEGM}(1,1)$ model, both in-sample and out-of-sample, has the smallest mean relative percentage error (MRPE). Specifically, the values of MRSPE and MRPPE are $1.9780 \%$ and $2.8027 \%$, respectively.

To show a direct comparison of the performances of $\operatorname{NEGM}(1,1), \operatorname{SAIGM}$, and $\operatorname{GM}(1,1)$, the scatter diagrams 
TABLE 1: Simulation/prediction values and errors of $\operatorname{NEGM}(1,1), \operatorname{SAIGM}$, and $\operatorname{GM}(1,1)$.

\begin{tabular}{|c|c|c|c|c|c|c|c|}
\hline \multirow{2}{*}{ Year } & \multirow{2}{*}{ Actual value $x^{(0)}(k)$} & \multicolumn{2}{|c|}{$\operatorname{NEGM}(1,1)$} & \multicolumn{2}{|c|}{ SAIGM } & \multicolumn{2}{|c|}{$\mathrm{GM}(1,1)$} \\
\hline & & $\widehat{x}^{(0)}(k)$ & $\Delta_{S}(k) \%$ & $\widehat{x}^{(0)}(k)$ & $\Delta_{S}(k) \%$ & $\widehat{x}^{(0)}(k)$ & $\Delta_{S}(k) \%$ \\
\hline 2000 & 1347.2 & 1347.2 & - & 1347.2 & - & 1347.2 & - \\
\hline 2001 & 1463.4 & 1463.4 & - & 1348.2 & 7.8687 & 1785.5 & 22.0076 \\
\hline 2002 & 1633.2 & 1573.9 & 3.6320 & 1642.6 & 0.5776 & 1955.0 & 19.7047 \\
\hline 2003 & 1903.2 & 1893.7 & 0.5007 & 1942.5 & 2.0632 & 2140.7 & 12.4777 \\
\hline 2004 & 2197.1 & 2216.4 & 0.8757 & 2247.7 & 2.3045 & 2344.0 & 6.6845 \\
\hline 2005 & 2494.0 & 2542.1 & 1.9260 & 2558.5 & 2.5873 & 2466.6 & 2.9095 \\
\hline 2006 & 2858.9 & 2870.9 & 0.4156 & 2874.9 & 0.5614 & 2810.3 & 1.7001 \\
\hline 2007 & 3271.2 & 3202.8 & 2.0968 & 3197.1 & 2.2651 & 3077.2 & 5.9312 \\
\hline 2008 & 3454.1 & 3537.8 & 2.4160 & 3525.1 & 2.0553 & 3369.4 & 2.4520 \\
\hline 2009 & 3703.2 & 3875.9 & 4.6563 & 3859.0 & 4.2077 & 3689.4 & 0.3732 \\
\hline 2010 & 4193.5 & 4217.3 & 0.5576 & 4199.0 & 0.1311 & 4039.7 & 3.6665 \\
\hline 2011 & 4700.1 & 4561.8 & 2.9524 & 4545.1 & 3.2971 & 4423.4 & 5.8875 \\
\hline 2012 & 4976.3 & 4909.5 & 1.3522 & 4897.5 & 1.5828 & 4843.5 & 2.6696 \\
\hline 2013 & 5420.3 & 5259.6 & 2.9583 & 5256.3 & 3.0252 & 5303.4 & 2.1565 \\
\hline 2014 & 5638.4 & 5614.9 & 0.4296 & 5621.6 & 0.2978 & 5807.1 & 2.9911 \\
\hline 2015 & 5802.0 & 5972.6 & 2.9252 & 5993.5 & 3.3007 & 6358.5 & 9.5919 \\
\hline $\operatorname{MRSPE}\left(\bar{\Delta}_{S} \%\right)$ & & & 1.9780 & & 2.4089 & & 6.7469 \\
\hline \multicolumn{8}{|c|}{ In-sample (simulation) } \\
\hline & $x^{(0)}(k)$ & $\hat{x}^{(0)}(k)$ & $\Delta_{P}(k) \%$ & $\hat{x}^{(0)}(k)$ & $\Delta_{P}(k) \%$ & $\widehat{x}^{(0)}(k)$ & $\Delta_{P}(k) \%$ \\
\hline 2016 & 6192.7 & 6333.6 & 2.2596 & 6372.1 & 2.8977 & 6962.4 & 12.4292 \\
\hline 2017 & 6482.1 & 6698.0 & 3.3149 & 6757.6 & 4.2507 & 7623.5 & 17.6085 \\
\hline $\operatorname{MRPPE}\left(\bar{\Delta}_{P} \%\right)$ & & & 2.8027 & & 3.5742 & & 15.0188 \\
\hline \multicolumn{8}{|c|}{ Out-of-sample (prediction) } \\
\hline CMRPE $\left(\frac{1}{\Delta} \%\right)$ & & & 2.3904 & & 2.9916 & & 10.8829 \\
\hline
\end{tabular}

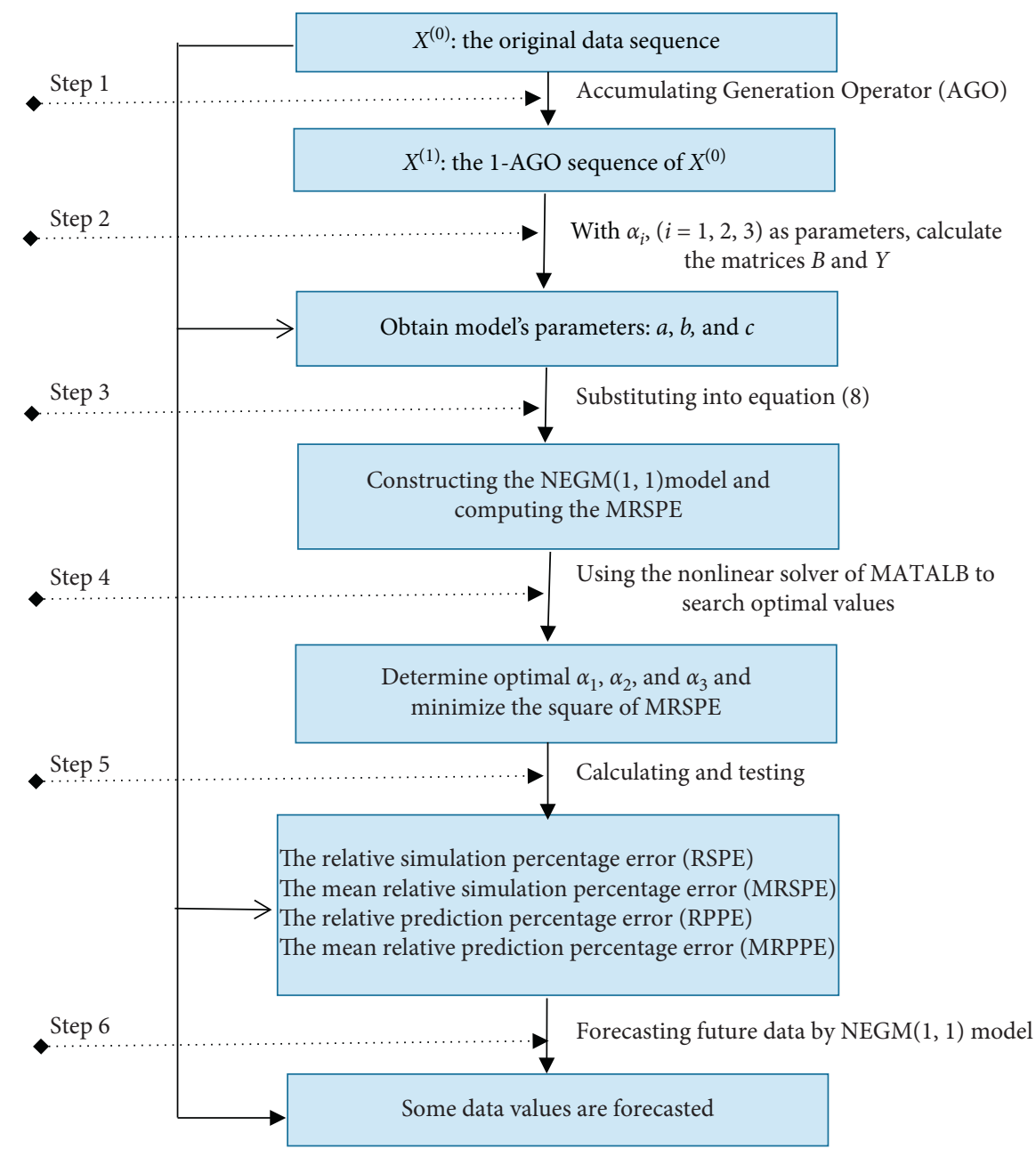

FIgure 1: The modeling flowchart of the $\operatorname{NEGM}(1,1)$ model. 
TABLE 2: China's electricity consumption from 2000 to 2017 (unit: billion kilowatt-hours).

\begin{tabular}{|c|c|c|c|c|c|c|c|c|c|}
\hline Year & 2000 & 2001 & 2002 & 2003 & 2004 & 2005 & 2006 & 2007 & 2008 \\
\hline Electricity consumption & 1347.2 & 1463.4 & 1633.2 & 1903.2 & 2197.1 & 2494 & 2858.9 & 3271.2 & 3454.1 \\
\hline Year & 2009 & 2010 & 2011 & 2012 & 2013 & 2014 & 2015 & 2016 & 2017 \\
\hline Electricity consumption & 3703.2 & 4193.5 & 4700.1 & 4976.3 & 5420.3 & 5638.4 & 5802.0 & 6192.7 & 6482.1 \\
\hline
\end{tabular}

Source: National Bureau of Statistics of China [1].

TABle 3: Parameter values for the $\operatorname{NEGM}(1,1)$ model.

\begin{tabular}{lcccccc}
\hline Parameter & $\alpha_{1}$ & $\alpha_{2}$ & $\alpha_{3}$ & $a$ & $b$ & $c$ \\
\hline Value & 0.0610 & 0.4810 & 0.4580 & -0.0093 & 303.7831 & 630.3808 \\
\hline
\end{tabular}

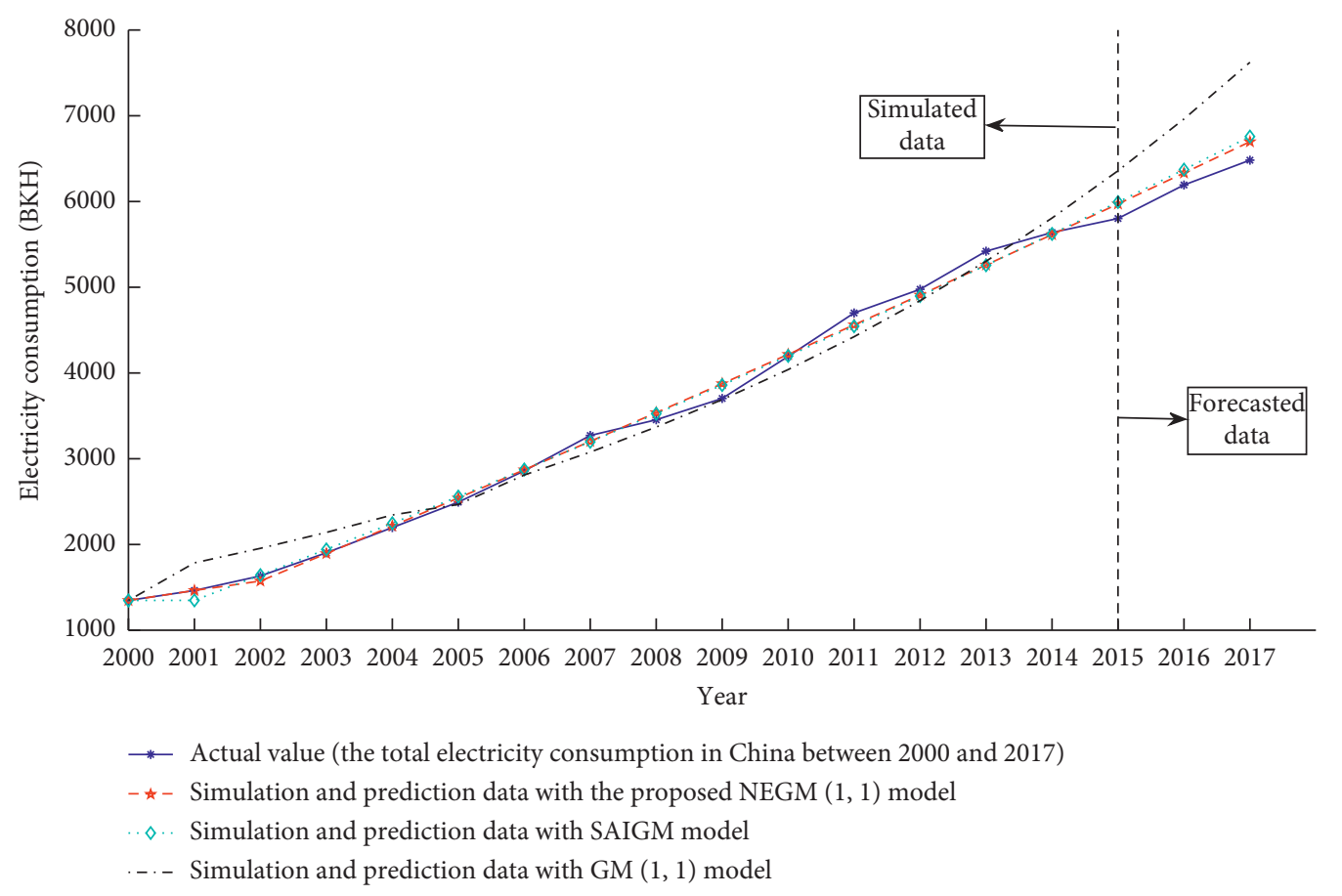

Figure 2: Simulation and prediction values of China's total electricity consumption.

with the simulation and prediction results and percentage errors of the three models in Table 1 are shown in Figures 2 and 3 , respectively.

According to 2 and 3 , it is easy to observe that the simulation-prediction curve of $\operatorname{NEGM}(1,1)$ is the closest to the real data, which indicates that the new model has the best comprehensive performance among the above three models. Furthermore, the comprehensive mean relative percentage error (CMRPE) is only $2.32 \%$, which is close to grade I of the grey model error level reference table [16]. Therefore, $\operatorname{NEGM}(1,1)$ is more suitable for forecasting the total electricity consumption in China.

Step 4. Prediction.

According to equation (20), the total electricity consumption in China from 2018 to 2025 can be predicted, as shown in Table 4.

From Table 4, we can see that in 2025, China's total electricity consumption is expected to reach 9736.9 (billion kw/h). Overall, China's total electricity consumption shows an increasing trend year by year in the next eight years.

\section{Suggestions}

According to the prediction results in Table 4, though the annual average growth rate of total electricity consumption in China is about $4.79 \%$, the total amount is still large. It is a major concern of China that some measures need to be taken to ensure the effective supply of electricity and rapid development of the following aspects.

Firstly, in terms of technology, China has continuously optimized the structure of the industry and controlled the excessive growth trend of industries with high energy consumption and high pollution. Simultaneously, it has promoted the development of high-tech industries and increased the research of electricity storage core technologies.

Secondly, in terms of policies, the power company has continuously accelerated the research and implementation 


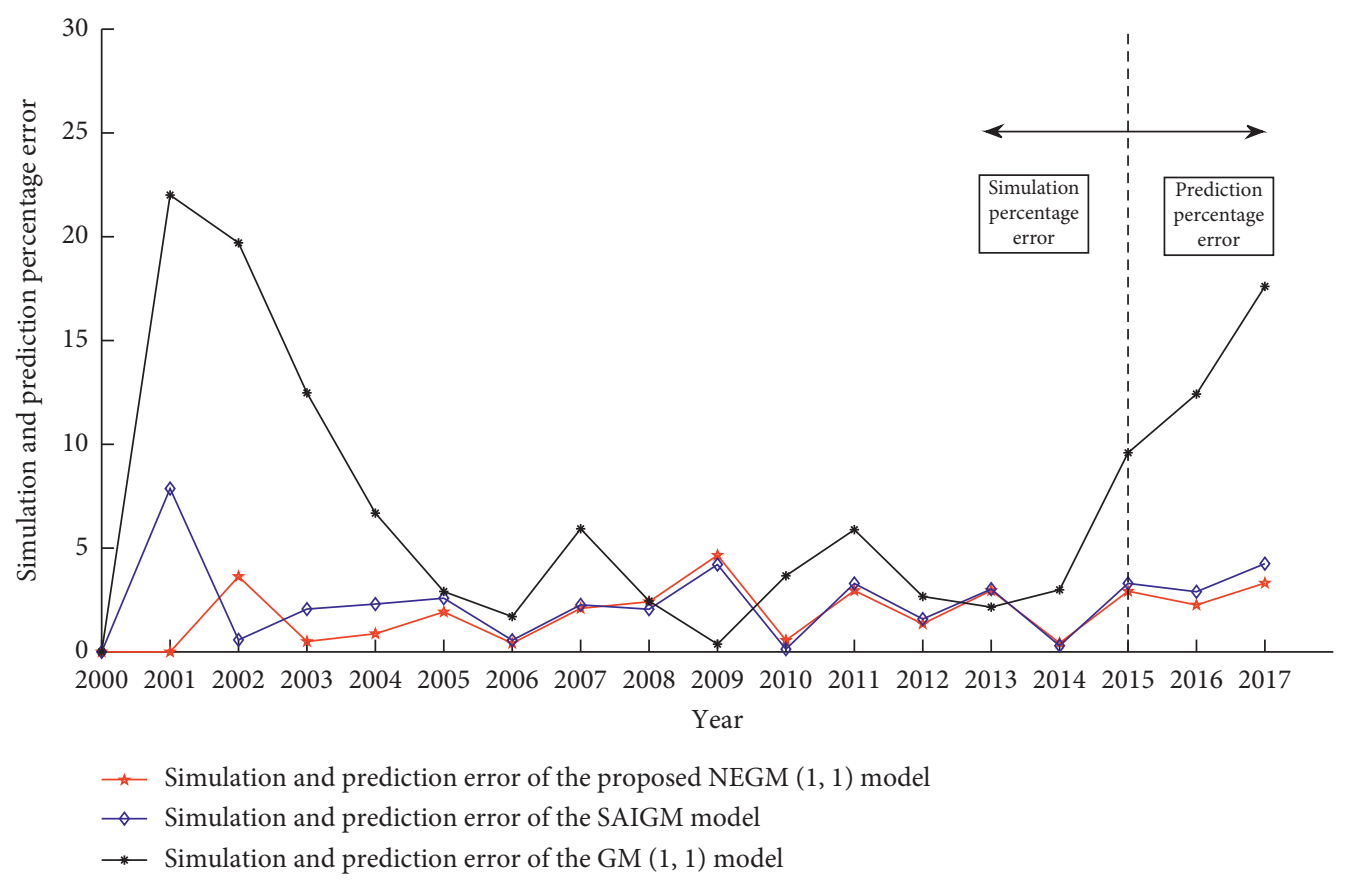

Figure 3: Simulation and prediction percentage errors of China's total electricity consumption.

TABle 4: Prediction data of the electricity consumption in China during 2018-2025 (unit: billion kilowatt-hours).

\begin{tabular}{lc}
\hline Year & Electricity consumption \\
\hline 2018 & 7064.6 \\
2019 & 7435.8 \\
2020 & 7810.4 \\
2021 & 8188.5 \\
2022 & 8570.2 \\
2023 & 8955.5 \\
2024 & 9344.4 \\
2025 & 9736.9 \\
\hline
\end{tabular}

of the renewable energy generation grid-connecting policy to promote the construction of microgrid facilities such as photovoltaic solar power. In addition, the electricity-saving awareness of residents should be cultivated, and it can employ the proper use of price leverage to strive for electricity conservation.

Thirdly, in terms of management, the Chinese government has continuously deepened the reform of power supply measurement according to market demands and scientifically promoted the construction of renewable energy. Moreover, it gradually established a management mechanism on energy storage for the power grid to increase the proportion of renewable energy in peaking alternative trading.

\section{Conclusion}

In order to weaken the influence of extreme values in the background value on the accuracy of forecasting models, a novel univariate grey model called $\operatorname{NEGM}(1,1)$ was proposed based on the SAIGM model, which adopted an extrapolation method with variable weights to calculate the background value. The new model was applied in forecasting China's total electricity consumption and compared with two other models, SAIGM and $\operatorname{GM}(1,1)$. Results showed that the $\operatorname{NEGM}(1,1)$ model outperformed the other models, which further verified that the new model enriched the theoretical framework of grey prediction models. Lastly, $\operatorname{NEGM}(1,1)$ was used to forecast the total electricity consumption in China from 2018 to 2025, and corresponding policy suggestions were given based on the prediction results.

Although the performance of the $\operatorname{NEGM}(1,1)$ model was better than that of SAIGM and GM $(1,1)$, there are still some limitations. Because $\operatorname{NEGM}(1,1)$ is a univariate model, the influence of external factors on China's total electricity consumption is not modeled. Therefore, by fully mining the ways of these factors affecting total electricity consumption and expanding $\operatorname{NEGM}(1,1)$ to a multivariate model, the performance of prediction may be further improved. The establishment of a multivariable $\operatorname{NEGM}(1, \mathrm{~N})$ model is our next research direction.

\section{Data Availability}

The data used to support the findings of this study are available from the corresponding author upon request.

\section{Conflicts of Interest}

The author declares that there are no conflicts of interest.

\section{Acknowledgments}

This study was supported by the National Social Science Foundation of China (no. 17ZDA065), the Natural Science 
Foundation of Chongqing Municipal Education Commission, China (no. KJQN201901601), and the Science and Technology Research Project of Chongqing University of Education (no. KY201910B).

\section{References}

[1] National Bureau of Statistics, http://www.stats.gov.cn/tjsj/ ndsj/.

[2] N. Amjady, "Short-term hourly load forecasting using timeseries modeling with peak load estimation capability," IEEE Transactions on Power Systems, vol. 16, no. 4, pp. 798-805, 2001.

[3] V. Bianco, O. Manca, and S. Nardini, "Electricity consumption forecasting in Italy using linear regression models," Energy, vol. 34, no. 9, pp. 1413-1421, 2009.

[4] Y. Wang, J. Wang, G. Zhao, and Y. Dong, "Application of residual modification approach in seasonal ARIMA for electricity demand forecasting: a case study of China," Energy Policy, vol. 48, pp. 284-294, 2012.

[5] M. H. Amini, A. Kargarian, and O. Karabasoglu, "ARIMAbased decoupled time series forecasting of electric vehicle charging demand for stochastic power system operation," Electric Power Systems Research, vol. 140, pp. 378-390, 2016.

[6] C. Li, S. Lin, F. Xu, D. Liu, and J. Liu, "Short-term wind power prediction based on data mining technology and improved support vector machine method: a case study in Northwest China," Journal of Cleaner Production, vol. 205, pp. 909-922, 2018.

[7] J. Che and J. Wang, "Short-term load forecasting using a kernel-based support vector regression combination model," Applied Energy, vol. 132, pp. 602-609, 2014.

[8] F. L. C. da Silva, F. L. Cyrino Oliveira, R. C. Souza, and R. C. Souza, "A bottom-up bayesian extension for long term electricity consumption forecasting," Energy, vol. 167, pp. 198-210, 2019.

[9] T. Ahmad and H. Chen, "Nonlinear autoregressive and random forest approaches to forecasting electricity load for utility energy management systems," Sustainable Cities and Society, vol. 45, pp. 460-473, 2019.

[10] Y. X. Wu and M. Xie, "Annual maximum load forecasting based on grey regression combined model of BP neural network," Southern Energy Construction, vol. 4, no. 2, pp. 46-50+57, 2017.

[11] K. Kandananond, "Forecasting electricity demand in Thailand with an artificial neural network approach," Energies, vol. 4, no. 8, pp. 1246-1257, 2011.

[12] Y. Long, Z. Y. Su, and Y. Wang, "Monthly load forecasting based on seasonal adjustment and BP neural network," Systems Engineering - Theory \& Practice, vol. 38, no. 4, pp. 1052-1060, 2018.

[13] W. Kong, Z. Y. Dong, Y. Jia, D. J. Hill, Y. Xu, and Y. Zhang, "Short-Term residential load forecasting based on LSTM recurrent neural network," IEEE Transactions on Smart Grid, vol. 10, no. 1, pp. 841-851, 2019.

[14] J. Lago, F. De Ridder, and B. De Schutter, "Forecasting spot electricity prices: deep learning approaches and empirical comparison of traditional algorithms," Applied Energy, vol. 221, pp. 386-405, 2018.

[15] J. L. Deng, "Control problems of grey systems," Systems \& Control Letters, vol. 1, no. 5, pp. 288-294, 1982.

[16] S. Liu, Y. Yang, and J. Forrest, Grey Data Analysis: Method, Models and Applications, Springer, pp. 45-65, Berlin, Germany.
[17] B. Zeng, H. Li, and X. Ma, "A novel multi-variable grey forecasting model and its application in forecasting the grain production in China," Computers \& Industrial Engineering, vol. 150, Article ID 106915, 2020.

[18] B. Zeng, X. Ma, and J. J. Shi, "A new-structure grey Verhulst model for China's tight gas production forecasting," Applied Soft Computing, vol. 96, Article ID 106600, 2020.

[19] B. Zeng, M. Zhou, X. Liu, and Z. Zhang, "Application of a new grey prediction model and grey average weakening buffer operator to forecast China's shale gas output," Energy Reports, vol. 6, pp. 1608-1618, 2020.

[20] B. Zeng and H. Li, "Prediction of coalbed methane production in China based on an optimized grey system model," Energy \& Fuels, vol. 35, no. 5, pp. 4333-4344, 2021.

[21] B. Zeng, H. Duan, Y. Bai, and W. Meng, "Forecasting the output of shale gas in China using an unbiased grey model and weakening buffer operator," Energy, vol. 151, pp. 238-249, 2018.

[22] Y. Wei and D.H. Hu, "Deficiency of the smoothness condition and its remedy," Systems Engineering - Theory \& Practice, vol. 29, no. 8, pp. 165-170, 2009.

[23] Y. Wang, Y. Dang, Y. Li, and S. Liu, “An approach to increase prediction precision of $\mathrm{GM}(1,1)$ model based on optimization of the initial condition," Expert Systems with Applications, vol. 37, no. 8, pp. 5640-5644, 2010.

[24] S. L. Li, B. Zeng, X. Ma, and D. H. Zhang, "A novel grey model with a three-parameter background value and its application in forecasting average annual water consumption per capita in urban areas along the Yangtze river basin," Journal of Grey System, vol. 32, no. 1, pp. 118-132, 2020.

[25] B. Zeng, M. Tong, and X. Ma, "A new-structure grey Verhulst model: development and performance comparison," Applied Mathematical Modelling, vol. 81, pp. 522-537, 2020.

[26] B. Zeng, H. Duan, and Y. Zhou, "A new multivariable grey prediction model with structure compatibility," Applied Mathematical Modelling, vol. 75, pp. 385-397, 2019.

[27] Y. Yang, Y. H. Chen, J. Shi, M. F. Liu, C. H. Li, and L. Li, “An improved grey neural network forecasting method based on genetic algorithm for oil consumption of China," Journal of Renewable and Sustainable Energy, vol. 8, pp. 332-343, 2016.

[28] Y.-C. Hu, P. Jiang, and P.-C. Lee, "Forecasting tourism demand by incorporating neural networks into Grey-Markov models," Journal of the Operational Research Society, vol. 70, no. 1, pp. 12-20, 2019.

[29] Y. Wang, Q. Liu, J. Tang, W. Cao, and X. Li, “Optimization approach of background value and initial item for improving prediction precision of $\operatorname{GM}(1,1)$ model," Journal of Systems Engineering and Electronics, vol. 25, no. 1, pp. 77-82, 2014.

[30] X. P. Xiao and H. H. Wang, "Change of GM $(1,1, \alpha)$ model background value on the influences of relative error," Systems Engineering - Theory \& Practice, vol. 34, no. 2, pp. 408-415, 2014.

[31] S. Q. Jiang, S. F. Liu, and X. C. Zhou, "Optimization of background value in GM $(1,1)$ based on compound trapezoid formula," Control and Decision, vol. 29, pp. 2221-2225, 2014.

[32] X. J. Wang, S. L. Yang, J. Ding, and H. J. Wang, "Dynamic $\operatorname{GM}(1,1)$ model based on cubic spline for electricity consumption prediction in smart grid," China Communications, vol. 7, pp. 83-88, 2010.

[33] B. Zeng and C. Luo, "Forecasting the total energy consumption in China using a new-structure grey system model," Grey Systems: Theory and Application, vol. 7, no. 2, pp. 194217, 2017. 
[34] B. Zeng, W. Meng, and M. Tong, "A self-adaptive intelligence grey predictive model with alterable structure and its application," Engineering Applications of Artificial Intelligence, vol. 50, pp. 236-244, 2016.

[35] J. Cui, Y. G. Dang, and S. F. Liu, "Novel grey forecasting model and its modeling mechanism," Control and Decision, vol. 24, no. 11, pp. 1702-1706, 2009. 